\title{
FILTRAGEM ADAPTATIVA PARA A ESTIMAÇÃO DA FREQÜÊNCIA EM SISTEMAS ELÉTRICOS DE POTÊNCIA
}

\author{
Daniel Barbosa* \\ dbarbosalsel.eesc.usp.br \\ Denis V. Coury* \\ couryesel.eesc.usp.br
}

\author{
Renato M. Monaro* \\ rmonarodsel.eesc.usp.br \\ Mário Oleskovicz* \\ oleskesel.eesc.usp.br
}

\author{
${ }^{*}$ SEL - EESC - USP \\ Av. Trabalhador São-Carlense, 400 \\ São Carlos, São Paulo, Brasil
}

\begin{abstract}
This research presents a method for frequency estimation in power system using adaptive filter based on the Least Mean Square algorithm (LMS). In power system analysis, the three-phase voltages are converted to a complex signal with the application of $\alpha \beta$-transform whose complex form was submitted to the algorithm of adaptive filtering. The computational simulations were accomplished using the software ATP. This utilization had as objective to generate data for the most severe and different situations for the verification and analysis of the proposed methodology. The results were to those of a commercial relay for validation, showing the advantages of the new method.
\end{abstract}

KEYWORDS: frequency estimation, adaptive filter, digital protection, power system, least mean square.

\section{RESUMO}

Esta pesquisa apresenta um método para a estimação da freqüência em sistemas elétricos de potência utilizando filtros adaptativos baseados no método dos mínimos quadrados (MMQ). A análise do sistema de potência é realizada através

\footnotetext{
Artigo submetido em 14/04/2007

1a. Revisão em 03/06/2007

2a. Revisão em 25/01/2008

3a. Revisão em 16/04/2008

Aceito sob recomendação do Editor Associado Prof. Carlos A. Castro
}

da conversão das tensões trifásicas em um sinal complexo pela aplicação da transformada $\alpha \beta$, sendo este direcionado ao algoritmo de filtragem adaptativa. As simulações computacionais, assim como a modelagem dos equipamentos, foram realizadas utilizando-se do software ATP (Alternative Transients Program). Este teve por objetivo, gerar dados das mais diversas e distintas situações para a verificação e análise da metodologia proposta, em comparação a resultados obtidos de um determinado relé comercial, habilitado à supervisão da freqüência do sistema.

PALAVRAS-CHAVE: estimação da frequiência, filtro adaptativo, proteção digital, sistema elétrico de potência, método dos mínimos quadrados (MMQ).

\section{INTRODUÇÃo}

Para proteção dos Sistemas Elétricos de Potência (SEPs) é fundamental a rápida e precisa detecção de anomalias, ou seja, a indicação de situações faltosas ou de condições anormais, para que estas possam ser rapidamente eliminadas, buscando a volta às condições normais de operação.

Com este objetivo, os relés de proteção monitoram constantemente parâmetros dos sinais trifásicos de tensão e corrente, incluindo a freqüência. Esta é uma das principais grandezas a ser observada em um SEP, já que, durante uma situação faltosa ou indesejada, sofrerá alterações incontestáveis. 
Como prática, admite-se uma faixa de variação na freqüência para a operação do sistema, estabelecida entre $60 \pm 0,5 \mathrm{~Hz}$ (ONS, 2001). Variações entre estes limites são constantemente observadas como conseqüência do balanço dinâmico entre a geração e a carga, cuja característica é variante no tempo. No entanto, variações maiores que as estabelecidas podem indicar situações de faltas ou de sobrecarga do sistema. Neste último caso, por exemplo, o relé de freqüência pode auxiliar na decisão de rejeição de cargas (Concordia et al., 1995).

Neste contexto, verifica-se a importância da freqüência para o SEP, especialmente se esta não respeitar os limites estabelecidos para a sua operação normal. Isto pode acarretar sérios problemas ao funcionamento dos equipamentos conectados a rede elétrica, tais como bancos de capacitores, geradores, motores e linhas de transmissão, comprometendo desta forma, o equilíbrio do SEP como um todo.

Faz-se necessário ressaltar a importância da proteção para a manutenção da operabilidade do sistema elétrico frente aos diversos distúrbios e situações transitórias que distorcem as características dos sinais analisados e comprometem a eficiência de operação do mesmo.

Especificamente, para a supervisão da freqüência do sistema sob determinada condição de operação, faz-se uso de relés de frequiência digitais, que acionam os dispositivos de proteção quando detectadas condições de oscilações da rede fora dos patamares aceitáveis sob o ponto de vista de operação normal do SEP.

Deve ainda ser lembrado que com o avanço tecnológico das últimas décadas e o aumento considerável da utilização de sistemas eletrônicos em equipamentos e máquinas, intensificou-se a preocupação com as variações de frequiência no sistema, uma vez que estes modernos componentes são mais sensíveis a este tipo de perturbação (Emanuel et al., 1991). Deste modo, observa-se que o aprimoramento das técnicas utilizadas para uma melhor estimação da flutuação da freqüência em um SEP é muito importante para a operação correta do mesmo.

Tipicamente, abordagens à estimação da freqüência estão associadas à proteção contra a perda de sincronismo, priorizando a manutenção da estabilidade do SEP. Nesse intuito, várias técnicas foram desenvolvidas, sendo principalmente baseadas na disposição dos fasores, no método dos mínimos quadrados, na Transformada Rápida de Fourier - FFT, em técnicas inteligentes e no filtro de Kalman (Phadke et al., 1983; Sachdev and Giray, 1985; Dash et al., 1999; Dash et al., 1997; Girgis and Ham, 1982). Cabe destacar neste contexto, o filtro adaptativo baseado no MMQ proposto por Pradkan (Pradhan et al., 2005). O MMQ foi introduzido inicialmente por Widrow e Hoff (Farhang-Boroujeny, 1999) para o processamento digital de sinais, sendo posteriormente amplamente utilizado devido a sua estrutura simplificada, eficiência e robustez computacional.

Nesta pesquisa, utiliza-se da forma complexa do MMQ (Widrow et al., 1975) com variação do tamanho de passo (Aboulnasr and Mayyas, 1997), o que possibilita maior velocidade de convergência, uma vez que o sinal complexo analisado é formado pelas tensões trifásicas do SEP préprocessadas pela transformada $\alpha \beta$.

Cabe adiantar que as simulações computacionais foram realizadas através do software ATP (EEUG, 1987). Por este, foram modelados diversos equipamentos que constituem um SEP, entre os quais, destacam-se: um gerador síncrono com controle de velocidade, parâmetros das linhas de transmissão dependentes da freqüência e os transformadores com as respectivas curvas de saturação. Desta forma, situações extremas de operação foram permitidas com o intuito de verificar o comportamento da técnica proposta e validar os resultados observados.

\section{O ALGORITMO BASEADO NO MÉTODO DOS MÍNIMOS QUADRADOS (MMQ)}

O algoritmo baseado no método dos mínimos quadrados (MMQ), apresentado na figura 1, é uma combinação do processo adaptativo com a filtragem digital. Nesta figura, $\bar{u}(n-1)=\left[\begin{array}{llll}u(n-1) & u(n-2) & \ldots & u(n-M)\end{array}\right]$ é o vetor de $\mathrm{M}$ valores passados; $\bar{w}(n)=$ $\left[\begin{array}{llll}w_{1}(n) & w_{2}(n) & \ldots & w_{N}(n)\end{array}\right]^{T}$ o vetor com os coeficientes do filtro; $y(n)$ a saída do filtro e $e(n)$ o erro associado à aproximação do filtro.

O sinal de entrada do filtro pode ser estimado pela minimização do erro quadrático através da adequação dos coeficientes de $\bar{w}(n)$, que são ajustados recursivamente para obtenção de valores ótimos. A cada iteração, tais coeficientes são calculados por:

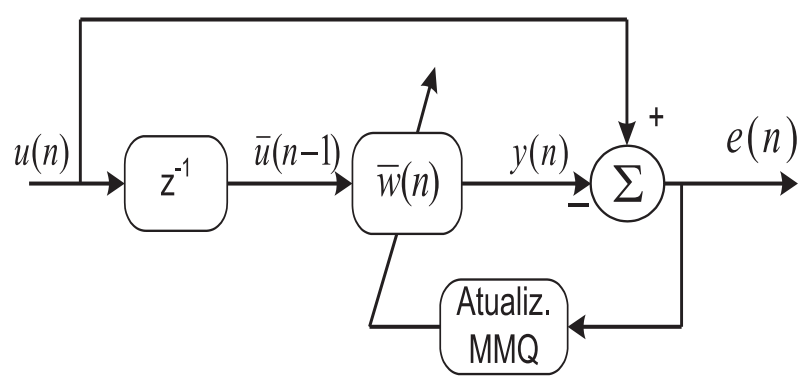

Figura 1: Filtro adaptativo utilizando o MMQ. 


$$
\bar{w}(n+1)=\bar{w}(n)+\mu(-\nabla(n))
$$

na qual $\mu$ é o parâmetro de convergência e $\nabla$ é o gradiente de desempenho flutuante do erro, sendo responsável por determinar o grau de ajuste necessário dos coeficientes.

O algoritmo MMQ é muito sensível à variação dos valores de $\mu$, sendo sua influência verificada na velocidade de estimação e no tempo de processamento tomado. Quanto menor o valor de $\mu$, maior será o tempo para se alcançar o erro desejado e vice-versa.

Contudo, é importante respeitar o intervalo de convergência dado por (Haykin, 2001):

$$
0<\mu<\frac{1}{N S_{\max }}
$$

Onde $N$ é o tamanho do filtro e $S_{\max }$ é valor máximo da densidade de potência espectral do sinal de entrada.

\section{O ALGORITMO ADAPTATIVO E A ESTI- MAÇÃO DA FREQÜÊNCIA}

Os filtros digitais fazem parte de conceituadas e consolidadas áreas de pesquisas, sendo, por exemplo, amplamente utilizados no processamento digital de sinais, onde o processo de filtragem é incorporado na maioria das aplicações.

Reportando-se à proteção digital, os filtros digitais possibilitam a extração das componentes de frequiência utilizadas nos algoritmos dos relés microprocessados, viabilizando a aplicação destes no monitoramento do sistema elétrico. As informações contidas nos dados coletados do sistema trifásico podem ser processadas simultaneamente, o que possibilitam resultados mais precisos que os obtidos com a aplicação de técnicas convencionais.

Ressalta-se que o algoritmo proposto, denominado de Algoritmo de Estimação da Frequiência pelo Método dos Mínimos Quadrados (AEFMMQ), parte da disponibilização de relés trifásicos. Em decorrência desta situação, todos os procedimentos da filtragem digital foram realizados para dados trifásicos previamente processados. Nesse sentido, as tensões aquisitadas da rede elétrica são representadas por:

$$
\begin{gathered}
V_{a}(n)=A_{\max } \cos (\omega n \Delta t+\phi)+\xi_{n_{a}} \\
V_{b}(n)=A_{\max } \cos \left(\omega n \Delta t+\phi-\frac{2 \pi}{3}\right)+\xi_{n_{b}} \\
V_{c}(n)=A_{\max } \cos \left(\omega n \Delta t+\phi+\frac{2 \pi}{3}\right)+\xi_{n_{c}}
\end{gathered}
$$

Nesta, $A_{\max }$ é a amplitude de pico da onda; $\omega$ é a freqüência angular do sinal ${ }^{1} ; n$ é o número da amostra do sinal discreto;

\footnotetext{
${ }^{1} \omega=2 \pi f$, sendo $f$ a frequiência do sistema
}

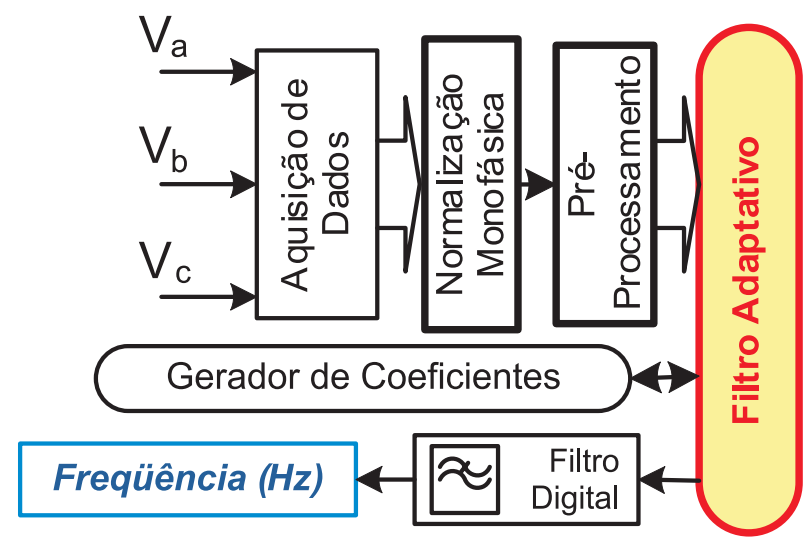

Figura 2: Diagrama de funcionamento básico do relé.

$\Delta t$ é o tempo entre duas amostras consecutivas; $\phi$ é fase do sinal e $\xi_{n}$ é o erro associado a cada amostra do sinal. A figura 2 ilustra o diagrama de funcionamento básico do relé proposto.

\subsection{Aquisição de Dados}

Todas as etapas de aquisição dos dados são efetuadas permitindo uma análise mais realista dos resultados obtidos. Os sinais de tensão de entrada simulados são caracterizados por uma alta taxa de amostragem, de maneira que possam representar mais fielmente os sinais analógicos do sistema elétrico em análise.

A figura 3 mostra o diagrama de aquisição de dados, evidenciando a utilização de filtros passa baixa Butterworth de segunda ordem (frequiência de corte em $200 \mathrm{~Hz}$ ), frequiência de amostragem igual a $1920 \mathrm{~Hz}$ e o conversor analógico digital (CAD) de 16 bits.

A aplicação do filtro passa baixa tem a finalidade de evitar o

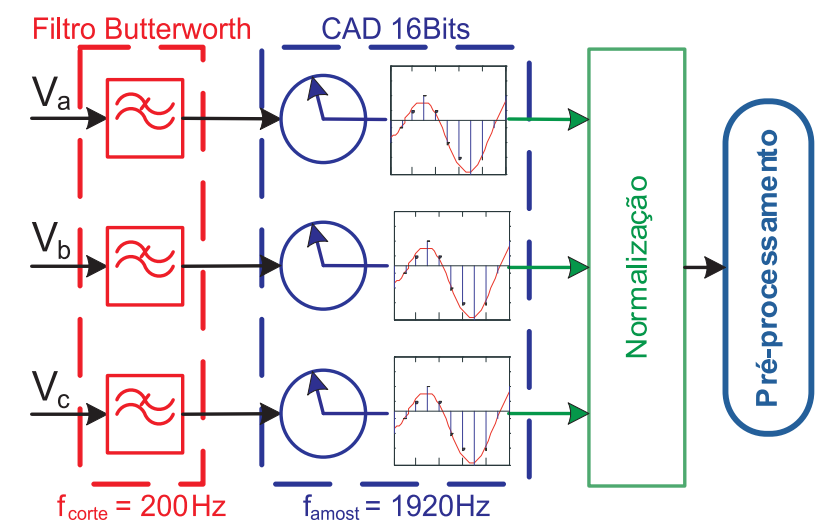

Figura 3: Fluxograma da aquisição de dados. 


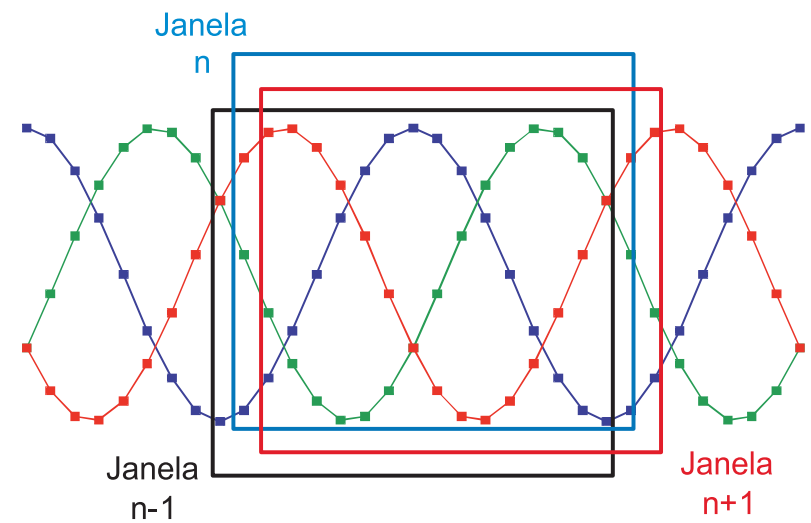

Figura 4: Progressão da janela a cada amostra nova.

espalhamento espectral e garantir que a representação digital após a conversão através do CAD seja fidedigna ao sinal de entrada. Vale comentar da baixa freqüência de corte $(200 \mathrm{~Hz})$ empregada, o que busca estabilizar e garantir a convergência do algoritmo MMQ implementado. Por esta situação, tem-se que a maioria das componentes harmônicas são eliminadas, o que aumenta a precisão na estimação da freqüência pelo método proposto.

A aquisição de dados é realizada de forma janelada e com passo fixo de uma amostra. Todo o processamento de filtragem que segue deverá ser realizado sobre uma janela de dados, respeitando-se o tempo hábil disponível para o processamento, que será quantificado pela disponibilização de uma nova amostra. A figura 4 ilustra o movimento da janela de dados a cada nova amostra disponibilizada.

\subsection{Normalização}

A normalização vem a padronizar os dados por fase obtidos do sistema elétrico, independentemente do nível de tensão analisado. Desta forma, caso ocorra um afundamento ou uma elevação em alguma(s) fase(s) do SEP, o algoritmo manterá sua estimação sem perda da precisão ou da velocidade de processamento. A figura 5 ilustra o procedimento de normalização dos dados coletados do SEP.

\subsection{Pré-processamento}

Quando da normalização dos dados obtidos do sistema, realiza-se um pré-processamento das informações para a obtenção do sinal na forma complexa a ser direcionado ao filtro digital. Os dados resultantes deste processo são obtidos através da aplicação da transformada $\alpha \beta$ sobre as tensões trifásicas da rede elétrica, conforme apresentado a seguir (Akke, 1997):

$$
\left[\begin{array}{l}
V_{\alpha}(n) \\
V_{\beta}(n)
\end{array}\right]=\sqrt{\frac{2}{3}}\left[\begin{array}{ccc}
1 & -\frac{1}{2} & -\frac{1}{2} \\
0 & \frac{\sqrt{3}}{2} & -\frac{\sqrt{3}}{2}
\end{array}\right][V(n)]
$$

sendo $V(n)=\left[\begin{array}{lll}V_{a}(n) & V_{b}(n) & V_{c}(n)\end{array}\right]^{T}$.

Na literatura, a utilização da transformada modal tem o objetivo desacoplar as fases do sistema trifásico, de modo que a rede seja tratada como três circuitos monofásicos e independentes, ou seja, possibilita a eliminação e/ou a redução do efeito da indutância mútua observadas nos sistemas elétricos de potência. Tal transformação é conhecida como transformada $\alpha \beta 0$ e inclui a componente de seqüência zero. Todavia, na aplicação apresentada nesse artigo, utiliza-se apenas as duas componentes perpendiculares, $\alpha$ e $\beta$, desconsiderando a componente de seqüência zero. Desta forma, observa-se que $V_{\alpha}$ e $V_{\beta}$ representam, somente, as tensões de seqüência positiva e negativa, bloqueando as principais componentes harmônicas homopolares (Akke, 1997).

Após o pré-processamento para a obtenção das componentes $\alpha$ e $\beta$ por 4 , estas são definidas como um sinal complexo, caracterizado por:

$$
u(n)=V_{\alpha}(n)+j V_{\beta}(n)
$$

\subsection{Gerador de Coeficientes}

A adaptação dos coeficientes do filtro adaptativo é simples e natural ao algoritmo, no qual o ajuste destes é realizado amostra a amostra de forma que o erro médio quadrático seja minimizado. Todavia, a fim de melhorar o desempenho do algoritmo e minimizar o tempo de processamento, inicializase os coeficientes do filtro da estimação atual, $\bar{w}(n)$, a partir do resultado da janela anterior. Em relação a primeira janela de dados, a estimativa é inicializada para a frequiência fundamental do sistema elétrico ${ }^{2}$. Tal procedimento tem o objetivo de aumentar a velocidade de inicialização do processamento em redes elétricas operando em condições normais.

\footnotetext{
${ }^{2}$ No sistema elétrico brasileiro, a freqüência nominal é de $60 \mathrm{~Hz}$
}

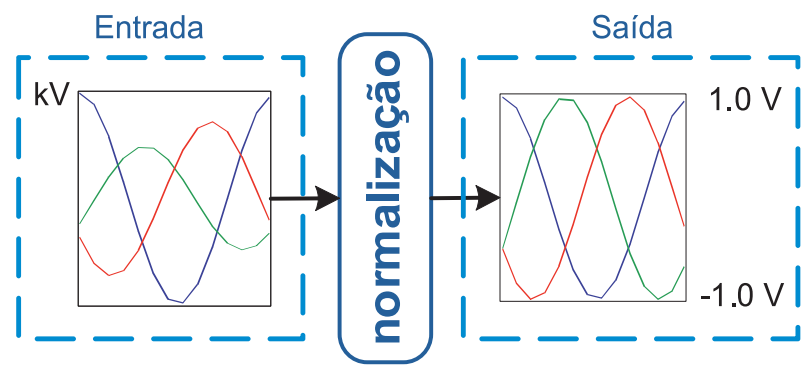

Figura 5: Fluxograma da normalização dos dados. 
Outra função exercida pelo Gerador de Coeficientes (GC) é o armazenamento dos coeficientes obtidos após a convergência do filtro para uma dada janela de dados, uma vez que estes serão utilizados na primeira iteração da próxima janela. Desta forma, evidencia-se uma relação entre o processamento atual e os posteriores.

\subsection{Filtro Adaptativo}

O filtro adaptativo corresponde à etapa do processamento efetivo do sinal, cujos coeficientes são atualizados recursivamente, proporcionando uma redução no erro quadrático e a convergência do mesmo. O sinal de erro é calculado em relação a diferença entre o valor desejado (sinal complexo de entrada) e o estimado, sendo neste caso dado por:

$$
e(n)=u(n)-y(n)
$$

Nesta, $y(n)$ representa o valor estimado, sendo calculado por:

$$
y(n)=\bar{w}^{H}(n) \bar{u}(n-1),
$$

na qual $H$ é a transformada de Hilbert.

É importante salientar que a tarefa do MMQ é apresentar o conjunto de coeficientes do filtro que minimize o erro, que será alcançado quando a saída do filtro for igual ao sinal de entrada. Nesse contexto, os coeficientes do filtro são atualizados a cada iteração até que o erro obtido esteja dentro do esperado. A alteração destes coeficientes é fornecida por (Widrow et al., 1975):

$$
\bar{w}(n+1)=\bar{w}(n)+\mu_{k} e_{k}^{*} \bar{u}(n-1)
$$

sendo que o símbolo $(*)$ denotado, representa o conjugado complexo e $\mu$ o fator de controle e razão da convergência do algoritmo.

Cabe comentar que nesta implementação, para melhorar a velocidade de convergência, caracterizou-se o algoritmo MMQ com um tamanho de passo variável. Desta maneira, a cada iteração, o parâmetro de convergência $(\mu)$ é modificado dentro de limites pré-estabelecidos (Aboulnasr and Mayyas, 1997). A atualização do $\mu$ é fornecida por:

$$
\mu_{k+1}=\lambda \mu_{k}+\gamma p_{k} p_{k}^{*}
$$

na qual $p_{k}$ retrata a autocorrelação dos erros. Após a atualização do $\mu$, verifica-se a inclusão deste na faixa de convergência pré-estabelecida, o que garante a estabilidade e velocidade do algoritmo frente a possíveis oscilações do sistema. A autocorrelação dos erros consecutivos é calculada por:

$$
p_{k}=\rho p_{k-1}+(1-\rho) e_{k} e_{k-1}
$$

$\mathrm{Na}$ equação, $\rho$ é o parâmetro de carregamento exponencial. As constantes $\rho(0<\rho<1), \lambda(0<\lambda<1)$ e $\gamma(0<\gamma)$ controlam o tempo de convergência e são determinadas por estudos estatísticos (Kwong and Johnston, 1992).

\subsection{Estimação da Freqüência}

A estimação da freqüência é efetuada conforme apresentada por (Begovic et al., 1993). Na busca pela diferença de fase, definiu-se a variável complexa $\Gamma$ como:

$$
\Gamma=y(n) y(n-1)^{*}
$$

A freqüência do sinal estimado $y(n)$ é calculada em função da diferença de fase entre duas iterações consecutivas, sendo esta fornecida pela equação abaixo:

$$
f_{\text {est }}=\frac{f_{s}}{2 \pi} \arctan \left(\frac{\Im(\Gamma)}{\Re(\Gamma)}\right),
$$

na qual $f_{\text {est }}$ é a freqüência estimada, $f_{s}$ é a freqüência de amostragem e $\Re()$ e $\Im()$ são as partes reais e imaginárias, respectivamente.

\subsection{Convergência}

Os critérios de parada adotados foram o número máximo de 1000 iterações e ou o erro absoluto inferior a $10^{-5}$. Tal estimativa de erro foi computada por:

$$
e_{\text {relat }}=a b s(y(n)-u(n)) \text {, }
$$

sendo $e_{\text {relat }}$ o erro relativo entre as amostras, $a b s()$ o valor absoluto, $y(n)$ o valor estimado e $u(n)$ o valor desejado ou amostra de entrada.

\subsection{Tratamento do sinal de saída}

O sinal de saída, ou seja, a freqüência estimada, passa por um filtro digital passa baixa Butterworth de segunda ordem com freqüência de corte em $5 \mathrm{~Hz}$. Esta situação proporciona a redução do efeito oscilatório característico da técnica empregada e de uma possível atuação errônea em situações de variação abrupta da freqüência do sinal. Cabe resaltar que o atraso proveniente da atuação do filtro passa baixa não compromete o desempenho do algoritmo, conforme pode ser observado nos resultados posteriores. 


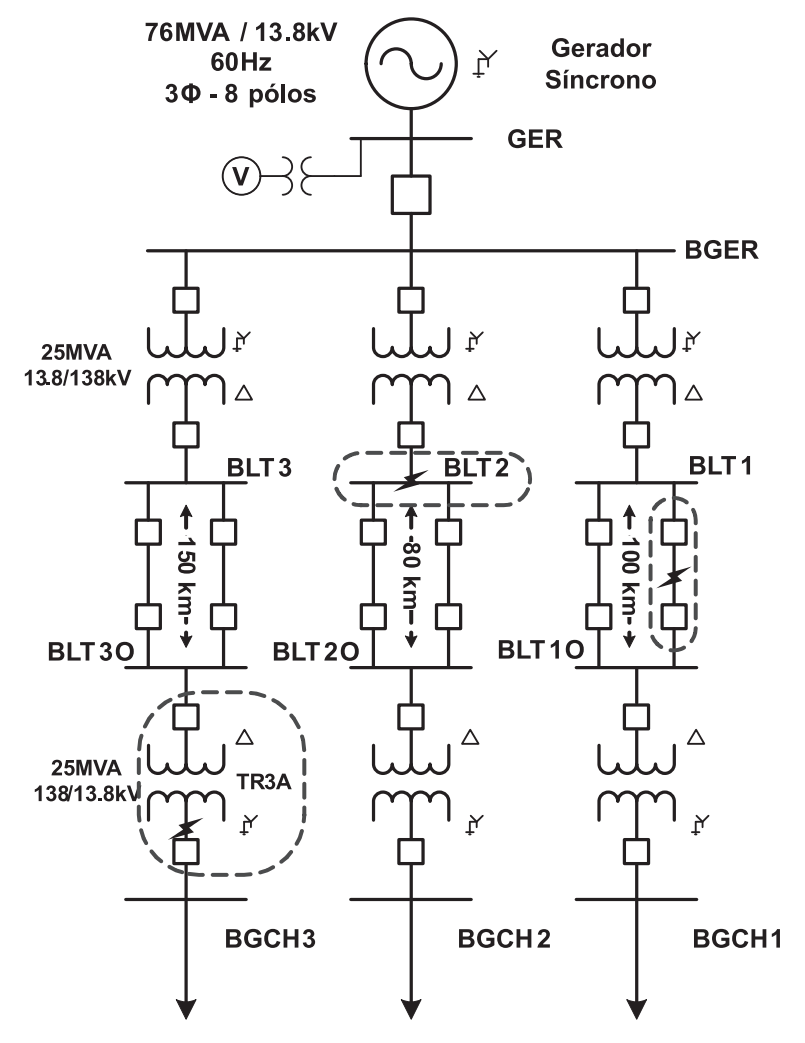

Figura 6: Representação do SEP analisado dispondo do software ATP.

\section{O SISTEMA ELÉTRICO ANALISADO}

A figura 6 mostra a representação do sistema elétrico utilizado nas simulações de manobras de cargas e faltas consideradas para avaliar o algoritmo de estimação da freqüência proposto.

O sistema elétrico é composto por um gerador síncrono de $13.8 \mathrm{kV}$ e potência aparente de 76MVA, transformadores elevadores com relações de $13.8 / 138 \mathrm{kV}$ e potência aparente de 25MVA, linhas de transmissão com extensões variando entre 80 e $150 \mathrm{~km}$, transformadores abaixadores similares aos elevadores e cargas caracterizadas por fator de potência de 0,92 indutivo e potência aparente variando entre 5 e 25 MVA. As tabelas 1 e 2 mostram os valores utilizados para caracterizar os diversos componentes via simulações computacionais dispondo do software ATP.

Na tabela 1, $S$ é a potência nominal, $N_{p}$ é o número de pólos, $V_{L}$ é a tensão nominal de linha, $f$ é a frequiência, $I F D$ é a corrente de campo, $R_{a}$ é a resistência de armadura, $X_{l}$ é a reatância de dispersão não saturada, $X_{o}$ é a reatância de seqüência zero não saturada, $X_{d}$ é a reatância síncrona de eixo direto não saturada, $X_{q}$ é a reatância síncrona de eixo em quadratura não saturada, $X_{d}^{\prime}$ é a reatância transitória de
Tabela 1: Dados do gerador síncrono utilizado na simulação.

\begin{tabular}{lclc}
\hline Descrição & Valor (un.) & Descrição & Valor (un.) \\
\hline \hline$S$ & $76(\mathrm{MVA})$ & $\left(N_{p}\right)$ & 8 \\
$V_{L}$ & $13,8\left(\mathrm{kV}_{r m s}\right)$ & $f$ & $60(\mathrm{~Hz})$ \\
$I F D$ & 250 (A) & $R_{a}$ & 0,004 (p.u.) \\
$X_{l}$ & 0,175 (p.u.) & $X_{o}$ & 0,132 (p.u.) \\
$X_{d}$ & 1,150 (p.u.) & $X_{q}$ & 0,685 (p.u.) \\
$X_{d}^{\prime}$ & 0,310 (p.u.) & $X_{d}^{\prime \prime}$ & 0,210 (p.u.) \\
$X_{q}^{\prime \prime}$ & 0,182 (p.u.) & $\tau_{d o}^{\prime}$ & 5,850 (seg.) \\
$\tau_{d o}^{\prime \prime}$ & 0,036 (seg.) & $\tau_{q o}^{\prime \prime}$ & 0,073 (seg.) \\
\hline
\end{tabular}

eixo direto não saturada, $X_{d}^{\prime \prime}$ é a reatância subtransitória de eixo direto não saturada, $X_{q}^{\prime \prime}$ é a reatância subtransitória de eixo em quadratura não saturada, $\tau_{d o}^{\prime}$ é a constante de tempo transitória em vazio de eixo direto, $\tau_{d o}^{\prime \prime}$ é a constante de tempo subtransitória em vazio de eixo direto e $\tau_{q o}^{\prime \prime}$ é a constante de tempo subtransitória em vazio de eixo em quadratura.

Tabela 2: Dados dos transformadores de potência.

\begin{tabular}{lcc}
\hline Elemento & $R_{+}(\Omega)$ & $L_{+}(m H)$ \\
\hline \hline Imped. primária do transf. abaixador & 1,7462 & 151,37 \\
Imped. secundária do transf. abaixador & 0,0175 & 1,514 \\
Imped. primária do transf. elevador & 0,0175 & 1,514 \\
Imped. secundária do transf. elevador & 1,7462 & 151,37 \\
\hline
\end{tabular}

É importante salientar que foi utilizado o modelo de linha de transmissão JMARTI vinculado ao software ATP, uma vez que este permite a variação dos parâmetros da linha em função da freqüência, possibilitando uma melhor representação do comportamento do sistema frente as perturbações provenientes de desequilíbrios entre geração e carga. A figura 7 ilustra o comportamento da impedância da linha de transmissão com a variação de carga.

Cabe ressaltar que o gerador síncrono foi caracterizado por uma conexão estrela aterrado, sendo o modelo utilizado via ATP o de uma máquina dinâmica síncrona trifásica, já que este considera os diversos parâmetros elétricos e mecânicos do gerador. Além do gerador, foi aplicado o controle de velocidade dinâmico para sistemas hidráulicos (Vieira Filho, 1984) para permitir uma melhor análise dos resultados, porém sem considerar o efeito da inércia da água. A equação 14 mostra a função de transferência do regulador de velocidade utilizado.

$$
\frac{\eta(s)}{\Delta F(s)}=-\frac{1}{R} \cdot \frac{1+s T_{r}}{\left(1+s T_{g}\right)\left(1+s \frac{r}{R} T_{r}\right)}
$$

Nesta, $\eta(s)$ é a posição do servo motor que aciona a turbina, 


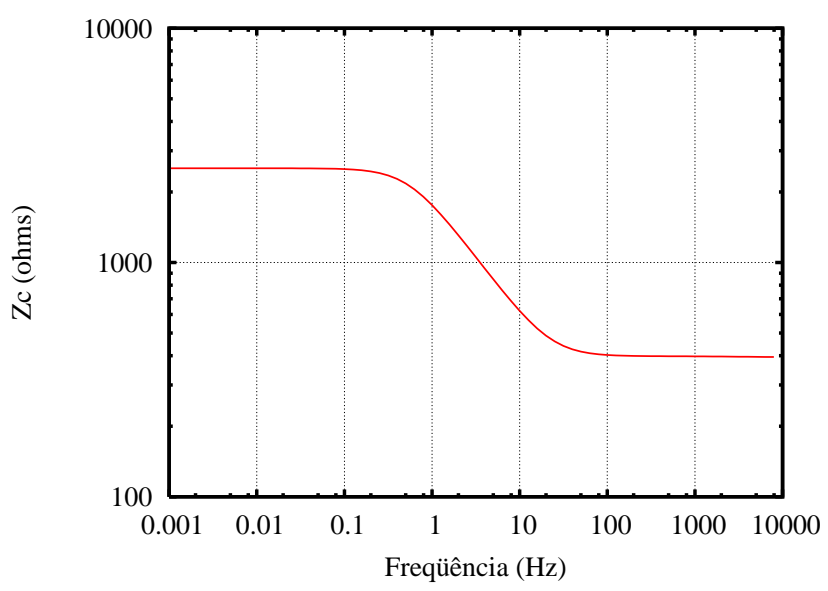

Figura 7: Comportamento da impedância da linha de transmissão em função da variação da carga.

$\Delta F(s))$ é o desvio de frequiência, $R$ é o estatismo permanente, $r$ é o estatismo transitório, $T_{g}$ é a constante de tempo do regulador de velocidade e $T_{t}$ é a constante associada ao estatismo transitório. A tabela 3 apresenta os valores dos parâmetros utilizados no regulador de velocidade.

Tabela 3: Parâmetros do regulador de velocidade.

\begin{tabular}{lc}
\hline Descrição & Valor $($ un.) \\
\hline \hline Constante de Tempo $\left(T_{g}\right)$ & $0,600(\mathrm{seg})$ \\
Constante de Tempo Dashpot $\left(T_{r}\right)$ & $0,838(\mathrm{seg})$ \\
Estatismo Transitório $(r)$ & 0,279 \\
Estatismo Permanente $(R)$ & 0,100 \\
Constante de Inércia do Gerador $(M)$ & $1,344(\mathrm{seg})$ \\
Constante de Tempo da Partida da Água $\left(T_{W}\right)$ & $0,150(\mathrm{seg})$ \\
\hline
\end{tabular}

\section{ANÁLISE DOS RESULTADOS}

A proposta dessa seção é apresentar alguns resultados da abordagem proposta em comparação às obtidas por um relé comercialmente disponível e a saída do software ATP, sem considerarmos o tempo de resposta dos métodos utilizados. Diversos e distintos testes foram realizados sobre o sistema anteriormente apresentado, dispondo de simulações pelo software ATP. Cabe comentar que a freqüência de amostragem empregada no AEFMMQ foi de $1920 \mathrm{~Hz}$ e que a oscilografia obtida do relé com a função 81 , além de conter 16 amostras por ciclo, apresenta-se filtrada.

É importante verificar que, devido a grande influência dos parâmetros de ajuste perante aos resultados apresentados, estes foram selecionados segundo (Kwong and Johnston, 1992). Os valores empregados são: $\mu_{\max }=0,18, \mu_{\min }=0,001$,

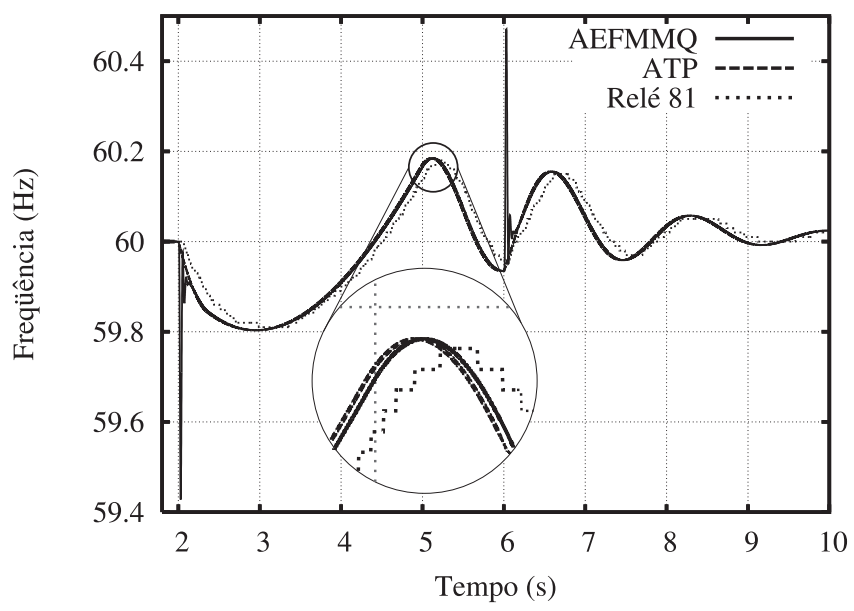

Figura 8: Ligamento e desligamento súbito do barramento $\mathrm{BGCH} 3$ em $2 \mathrm{~s}$ e $6 \mathrm{~s}$, respectivamente.

$p_{\text {inicial }}=0, \lambda=0,97, \gamma=0,01$ e $\rho=0,99$. Com base na figura 6 as situações avaliadas foram:

1. Entrada e saída súbita de um bloco de cargas;

2. Falta a $50 \%$ do comprimento da linha 1 ;

3. Falta envolvendo a fase $A$ com conexão a terra (AT) no barramento BLT2 à $0,5 \mathrm{~s}$;

4. Energização do transformador TR3A sob falta.

\subsection{Entrada e saída súbita de um bloco de cargas}

A figura 8 mostra a estimação da freqüência do gerador síncrono através do relé (81), do software ATP e do AEFMMQ para a entrada e saída do bloco de carga alimentado pelo barramento BGCH3. Nessa figura e em detalhe, também é possível observar um pequeno atraso na estimação da freqüência proporcionado pelo relé, quando comparada ao resultado do software ATP como referência. Nessa situação, verifica-se a precisão da estimação do AEFMMQ, que mesmo em pontos mais críticos consegue acompanhar o comportamento do sistema.

\subsection{Falta a $50 \%$ do comprimento da linha 1}

Na figura 9 é observada a variação da frequiência para uma falta AT à 50\% do comprimento da linha de transmissão 1 . Nessa situação, foi considerada as aberturas tripolares dos disjuntores correspondentes após $80 \mathrm{~ms}$ da aplicação da falta. 


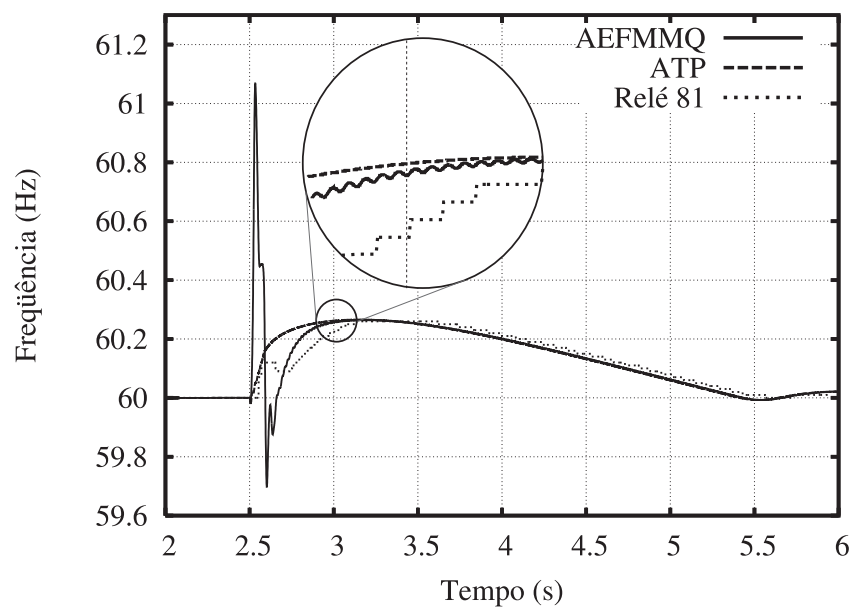

Figura 9: Falta $3 \phi$ a $50 \%$ do comprimento da linha 1 com posterior desligamento.

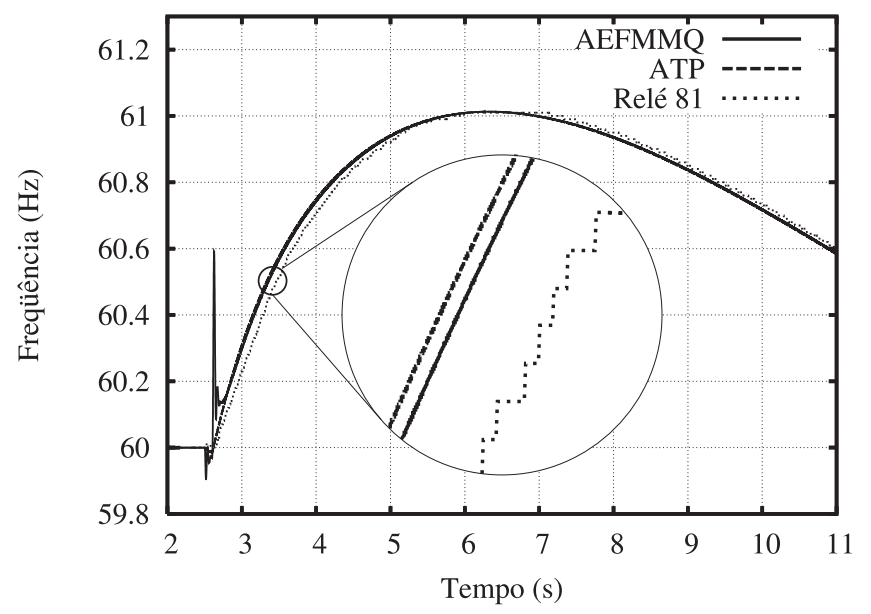

Figura 10: Falta AT em BLT2 à 2,5s e abertura do disjuntor em 2,6s

Pela figura 9, verifica-se a recuperação do sincronismo da máquina sem a necessidade da atuação dos relés, uma vez que para o sistema elétrico brasileiro a intervenção direta no controle automático de cargas deve acontecer quando a freqüência sair da faixa de $60 \pm 0,5 \mathrm{~Hz}$ (ONS, 2001).

\subsection{Falta AT no barramento BLT2 à 2,5s}

Na figura 10, tem-se a resposta da técnica proposta frente a uma situação de falta AT no barramento BLT2 à 2,5s com a consequiente abertura dos disjuntores associados em 2,6s.

É factível observar que apesar da retirada do defeito após $100 \mathrm{~ms}$ e da tendência de recuperação do sincronismo do gerador, a freqüência extrapola os limites de operação, o que acarretará em uma provável atuação do sistema de proteção

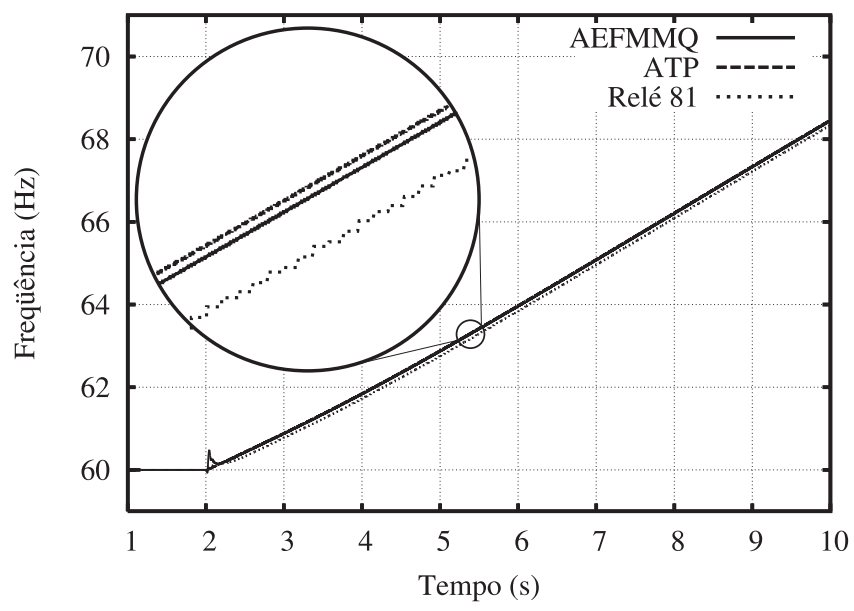

Figura 11: Energização do transformador TR3A sob falta externa

pelas técnicas apresentadas.

\subsection{Energização do transformador TR3A sob falta externa}

Pela figura 11, também se observam as respostas do relé (81) e do algoritmo proposto para uma situação de energização do transformador TR3A sob falta externa ao enrolamento secundário. A análise desta condição é de fundamental importância para se verificar a robustez do algoritmo frente às situações observadas em campo, principalmente devido aos altos índices de distorções apresentadas em situações desta natureza.

Observa-se para esse teste, que o relé (81) e o AEFMMQ apresentaram respostas praticamente idênticas, embora o relé apresente um pequeno atraso, possivelmente atribuído ao processo de filtragem do mesmo.

\section{CONCLUSÕES}

Este trabalho aponta um método alternativo para a estimação da frequiência em sistemas elétricos de potência, aplicandose o algoritmo MMQ. O emprego da filtragem digital e da transformada $\alpha \beta$ na técnica proposta possibilitaram a utilização simultânea e simplificada das tensões trifásicas da rede elétrica.

No algoritmo MMQ complexo considerado, empregou-se o ajuste do tamanho do passo como sendo variante no tempo com a estimação dos erros decorrentes.

As situações empregadas para o teste do algoritmo proposto foram obtidas por simulações computacionais através da utilização do software ATP, possibilitando características pecu- 
liares e observáveis nos parâmetros das formas de ondas trifásicas. A teoria de filtros adaptativos aplicada à proteção digital proporcionou resultados rápidos e confiáveis, além das seguintes constatações:

1. O algoritmo AEFMMQ pode ser aplicado à diversas situações e níveis de tensão, uma vez que este não é influenciado pela a amplitude das formas de onda analisadas.

2. Robustez à situações de falha do SEP, pois as três tensões do sistema são avaliadas e não apenas a fase A, como em alguns dos relés comerciais.

3. Facilidade de aplicação, já que a configuração é única, não necessitando de ajustes adicionais e conhecimento das demais funções do relé, como no caso do relé (81) utilizado.

Além destes três apontamentos, é importante ressaltar a facilidade de implementação e a eficiência computacional observada, induzindo à possíveis aplicações comerciais.

\section{AGRADECIMENTOS}

Os autores gostariam de agradecer ao Laboratório de Sistemas de Energia Elétrica (LSEE) da Escola de Engenharia Elétrica de São Carlos (EESC), Universidade de São Paulo (USP), pela infra-estrutura proporcionada no desenvolvimento deste trabalho, assim como ao Engenheiro Ulisses Chemin Netto pela valiosa contribuição, bem como ao apoio financeiro recebido por parte da CAPES (Coordenação de Aperfeiçoamento de Pessoal de Nível Superior) e da FAPESP (Fundação de Amparo a Pesquisa do Estado de São Paulo).

\section{REFERÊNCIAS}

Aboulnasr, T. and Mayyas, K. (1997). A robust variable stepsize LMS-type algorithm: Analysis and simulations, IEEE Transactions on Signal Processing 45(3): 631639.

Akke, M. (1997). Frequency estimation by demodulation of two complex signals, IEEE Transactions on Power Delivery 12(1): 157-163.

Begovic, M. M., Djuric, P. M., Dunlap, S. and Phadke, A. G. (1993). Frequency tracking in power networks in the presence of harmonics, IEEE Transactions on Power Delivery 8: 480-486.

Concordia, C., Fink, L. H. and Poullikkas, G. (1995). Load shedding on an isolated system, 10(3): 1467-1472.
Dash, P. K., Padhan, A. K. and Panda, G. (1999). Frequency estimation of distorted power system signals using extended complex Kalman filter, IEEE Transactions on Power Delivery 14(3): 761-766.

Dash, P. K., Swain, D. P., Routray, A. and Liew, A. C. (1997). An adaptive neural network approach for the estimation of power system frequency, Electric Power Systems Research 41: 203-210.

EEUG (1987). Alternative Transients Program Rule Book, LEC.

Emanuel, A. E., Orr, J. A., Cyganski, D. and Gulachenski, E. M. (1991). A survey of harmonic voltages and currents at distribution substations, IEEE Transactions on Power Delivery 6(4): 1883-1890.

Farhang-Boroujeny, B. (1999). Adaptive Filters:Theory and Applications, John Wiley \& Sons, Inc.

Girgis, A. A. and Ham, F. M. (1982). A new FFTbased digital frequency relay for load shedding, IEEE Transactions on Power Apparatus and Systems PAS101(2): 433-439.

Haykin, S. (2001). Adaptive Filter Theory, 4 edn, Prentice Hall, New Jersey.

Kwong, R. H. and Johnston, E. W. (1992). A variable step size LMS algorithm, IEEE Transactions on Signal Processing 40: 1633-1642.

ONS (2001). Submódulo 10.8 - Norma de Operação - Controle da Geração em Operação Normal, 2 edn, Operador Nacional do Sistema.

Phadke, A. G., Thorp, J. S. and Adamiak, M. G. (1983). A new measurement technique for tracking voltage phasors, local system frequency, and rate of change of frequency, IEEE Transactions on Power Apparatus and Systems PAS-102(5): 1025-1038.

Pradhan, A. K., Routray, A. and Basak, A. (2005). Power system frequency estimation using least mean square technique, IEEE Transactions on Power Delivery 20(3): 1812-1816.

Sachdev, M. S. and Giray, M. M. (1985). A least error squares technique for determining power system frequency, IEEE Transactions on Power Apparatus and Systems PAS-104(2): 437-444.

Vieira Filho, X. (1984). Operação de Sistemas de Potência com Controle Automático de Geração, Editora Campus Ltda, Rio de Janeiro.

Widrow, B., McCool, J. and Ball, M. (1975). The complex $1 \mathrm{~ms}$ algorithm, Proceedings of the IEEE 63: 719-720. 